\title{
There Are Integral Heptagons, no Three Points on a Line, no Four on a Circle
}

\author{
Tobias Kreisel · Sascha Kurz
}

Received: 6 October 2006 / Revised: 10 September 2007 /

Published online: 29 September 2007

(C) Springer Science+Business Media, LLC 2007

\begin{abstract}
We give two configurations of seven points in the plane, no three points in a line, no four points on a circle with pairwise integral distances. This answers a famous question of Paul Erdős.
\end{abstract}

Keywords Integral distances · Exhaustive search · Orderly generation · Solution to an Erdős problem

\section{Introduction}

A famous open problem of P. Erdôs asks for seven points in the plane, no three on a line, no four on a circle with pairwise rational or integral distances [1, 3]. For six points parameter solutions for infinite families of such point sets are known, see e.g. [6]. Since for finite point sets we can multiply the occurring distances with their denominators' smallest common multiple we confine ourselves to considering integral distances only. From the combinatorial point of view the question for the smallest possible diameter $\dot{d}(2, n)$ of $n$ points arises, where the diameter is the largest occurring distance in a point set. So far

$$
(\dot{d}(2, n))_{n=3, \ldots, 6}=1,8,73,174
$$

are known [4]. By exhaustive search the bound $\dot{d}(2,7) \geq 20000$ could be determined $[9,11]$. Up to diameter 20000 there are only few integral point sets consisting of 6 points, no three on a line, no four on a circle with pairwise integral distances, see

T. Kreisel $(\varangle) \cdot$ S. Kurz

Department of Mathematics, University of Bayreuth, 95440 Bayreuth, Germany

e-mail: tobias.kreisel@uni-bayreuth.de

S. Kurz

e-mail: sascha.kurz@uni-bayreuth.de 
[8] for a complete list. Some attempts to show that no integral point set in general position consisting of more than six points can exist are known [5], but the suggested proofs turned out to be incorrect. So there was little hope to discover such a point set. But then by a suggestion of S. Dimiev [2] we considered integral point sets over $\mathbb{Z}_{n}^{2}$ [7].

Definition 1 Two points $\left(u_{1}, \ldots, u_{m}\right),\left(v_{1}, \ldots, v_{m}\right) \in \mathbb{Z}_{n}^{m}:=(\mathbb{Z} \backslash \mathbb{Z} n)^{m}$ are at integral distance if there exists a number $d \in \mathbb{Z}_{n}$ with $\sum_{i=1}^{m}\left(u_{i}-v_{i}\right)^{2}=d^{2}$.

So, an integral point set in $\mathbb{Z}_{n}^{2}$ is defined as a subset of $\mathbb{Z}_{n}^{2}$ where all pairs of points are at integral distance. To have an analogue to the "no three on a line and no four on a circle" restriction we need two further definitions.

Definition 2 A set of $r$ points $\left(u_{i}, v_{i}\right) \in \mathbb{Z}_{n}^{2}$ is collinear if there are $a, b, t_{1}, t_{2}, w_{i} \in$ $\mathbb{Z}_{n}$ with $a+w_{i} t_{1}=u_{i}$ and $b+w_{i} t_{2}=v_{i}$.

Definition 3 Four points $p_{i}=\left(x_{i}, y_{i}\right)$ in $\mathbb{Z}_{n}^{2}$ are said to be situated on a circle if there exist $a, b \in \mathbb{Z}_{n}, r \in \mathbb{Z}_{n} \backslash\{\overline{0}\}$ with $\left(x_{i}-a\right)^{2}+\left(y_{i}-b\right)^{2}=r^{2} \forall i$.

By $\dot{\mathcal{I}}(n, 2)$ we denote the maximum number of points in $\mathbb{Z}_{n}^{2}$ with pairwise integral distances where no three are collinear and no four points are situated on a circle. By combinatorial search techniques - see [7] for the details-we found two point sets proving $\dot{\mathcal{I}}(50,2) \geq 12$ and $\dot{\mathcal{I}}(61,2) \geq 9$. Surely this does not imply the existence of an integral point set over the real plane in general position, i.e., no three points on a line, no four points on a circle; however, it did give us a fresh impetus to continue our search.

\section{Integral Heptagons in General Position}

The results for the "relaxed" problem over $\mathbb{Z}_{n}^{2}$ motivated us to maintain our approach of exhaustive generation of all plane integral point sets in general position up to a given diameter by a variant of orderly generation, see $[9,11]$ for details. Also, without changing our approach but simply by harnessing more computational power we were lucky enough to discover the following distance matrix

$\left(\begin{array}{ccccccc}0 & 22270 & 22098 & 16637 & 9248 & 8908 & 8636 \\ 22270 & 0 & 21488 & 11397 & 15138 & 20698 & 13746 \\ 22098 & 21488 & 0 & 10795 & 14450 & 13430 & 20066 \\ 16637 & 11397 & 10795 & 0 & 7395 & 11135 & 11049 \\ 9248 & 15138 & 14450 & 7395 & 0 & 5780 & 5916 \\ 8908 & 20698 & 13430 & 11135 & 5780 & 0 & 10744 \\ 8636 & 13746 & 20066 & 11049 & 5916 & 10744 & 0\end{array}\right)$

corresponding to a plane integral point set in general position with diameter 22270 consisting of seven points. So this answers Erdős's question positively. Since we applied an exhaustive search we received: 


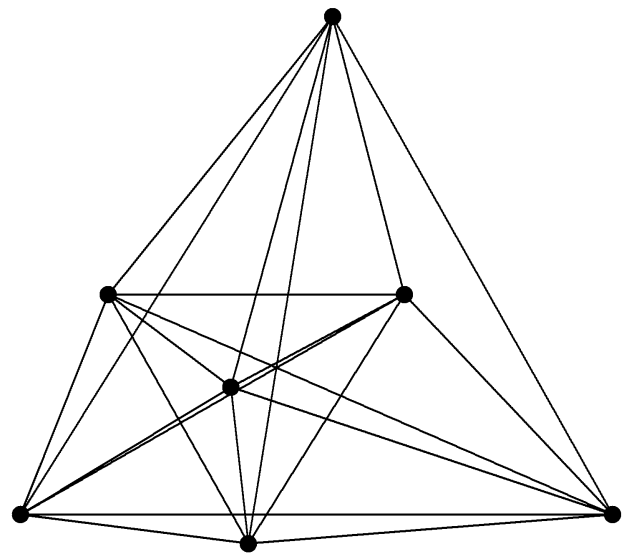

$$
\begin{aligned}
& \frac{0}{1}, \frac{0}{1} \sqrt{2002} \\
& \frac{22270}{1}, \frac{0}{1} \sqrt{2002} \\
& \frac{26127018}{2227}, \frac{932064}{2227} \sqrt{2002} \\
& \frac{245363}{17}, \frac{3144}{17} \sqrt{2002} \\
& \frac{17615968}{2227}, \frac{238464}{2227} \sqrt{2002} \\
& \frac{56068}{17}, \frac{3144}{17} \sqrt{2002} \\
& \frac{19079044}{2227},-\frac{54168}{2227} \sqrt{2002}
\end{aligned}
$$

Fig. 1 First example of an integral heptagon in general position

Theorem $1 \dot{d}(2,7)=22270$.

To avoid duplicated listings of isomorphic point sets we give all point sets in the following canonical form. Consider the vector $v(\Delta)$ formed by the columns of the upper right triangle of a distance matrix $\Delta$. A certain distance matrix $\Delta$ of a point set $\mathcal{P}$ (induced by a labeling of the points) is said to be canonical or maximal if its vector $v(\Delta)$ is the largest one in the set of all vectors of distance matrices of $\mathcal{P}$ with respect to the lexicographic order.

In Fig. 1 we give an embedding of distance matrix (1) in the plane and an exact coordinate representation. Discovering this point set clearly motivates to search for further examples to get ideas how to construct an infinite family of examples. Unfortunately, this point set is the only example with at most 30000 in diameter. For diameters greater than 30000 our approach of exhaustive search requires too much computational power so that we decided to skip to a restricted search. To describe the details of our restriction of the search space we need:

Definition 4 The characteristic of an integral triangle with side lengths $a, b, c \in \mathbb{Z}$ is the square free part of $(a+b+c)(a+b-c)(a-b+c)(-a+b+c)$.

Theorem 2 Each nondegenerated triangle in a plane integral point set has equal characteristic.

In point set (1) the characteristic is given by $2002=2 \cdot 7 \cdot 11 \cdot 13$ which explains the shape of the $y$-coordinates, see Fig. 1 and [10]. We notice that the characteristic of point set (1) is composed of relatively small prime factors. By a look at our list of integral hexagons in general position [8] we see that this seems to be a phenomenon that holds for a great part of the known examples. This phenomenon seems to hold for similar problems also. By determining the minimum diameter $d(2, n)$ of plane integral point sets without further restrictions up to $n=122$ points [11] we could check that the known minimal examples also have a characteristic composed of small prime 
factors. If additionally no three points are allowed to be collinear we denote the corresponding minimum diameter by $\bar{d}(n, 2)$. By determining all those minimal integral point sets with up to $n=36$ points $[9,11]$ we could check that the same phenomenon also occurs in this case. So it seemed worth a try to exhaustively construct all plane integral point sets in general position with given diameter of at most 70000 and the characteristic being a divisor of $6469693230=2 \cdot 3 \cdot 5 \cdot 7 \cdot 11 \cdot 13 \cdot 17 \cdot 19 \cdot 23 \cdot 29$. The outcome was yet another example:

$\left(\begin{array}{ccccccc}0 & 66810 & 66555 & 66294 & 49928 & 41238 & 40290 \\ 66810 & 0 & 32385 & 64464 & 32258 & 25908 & 52020 \\ 66555 & 32385 & 0 & 34191 & 16637 & 33147 & 33405 \\ 66294 & 64464 & 34191 & 0 & 34322 & 53244 & 26724 \\ 49928 & 32258 & 16637 & 34322 & 0 & 20066 & 20698 \\ 41238 & 25908 & 33147 & 53244 & 20066 & 0 & 32232 \\ 40290 & 52020 & 33405 & 26724 & 20698 & 32232 & 0\end{array}\right)$.

Unfortunately, the discovery of further examples is currently beyond our means, since the algorithm we use is of running time $\Omega\left(d^{3}\right)$ for the search for plane integral point sets in general position with diameter at most $d$. Though the restriction on the characteristic did accelerate computations significantly the theoretic lower bound for the complexity remains. (There are $O\left(d^{3}\right)$ integral triangles with diameter at most $d$.)

\section{Open Problems}

Clearly, one can ask for further examples or an infinite family of integral heptagons in general position. Since our two given examples are in nonconvex position it would be interesting to see a convex example. As a further restriction Bell and Noll [12] also required the coordinates of the point sets to be integral. Such point sets are commonly called $n_{m}$-clusters, where $n$ is the number of points and $m$ is the dimension. In general, the set of $n_{2}$-cluster equals the set of plane integral point sets in general position with characteristic 1 . So far no 72 -cluster is known and even its existence is unclear. The smallest $6_{2}$-cluster has diameter 1886 . At first sight it seems that we have answered Erdős question completely, but from a realistic point of view we have only pushed the frontier a step further. Originally P. Erdôs asked for five points in the plain, no three on a line, no four on a circle with pairwise integral distances. When such a set was found he asked for 6-set, then for a 7-set. So now we ask as a substitute:

"Are there eight points in the plane, no three on a line, no four on a circle with pairwise integral distances?"

\section{References}

1. Brass, P., Moser, W., Pach, J.: Research Problems in Discrete Geometry. Springer, Berlin (2005)

2. Dimiev, S.: A setting for a Diophantine distance geometry. Tensor (N.S.) 66(3), 275-283 (2005)

3. Guy, R.K.: Unsolved Problems in Number Theory, 2nd edn. Springer, Berlin (1994)

4. Harborth, H.: Integral distances in point sets. In: Butzer, P.L., et al. (eds.) Karl der Grosse und sein Nachwirken. 1200 Jahre Kultur und Wissenschaft in Europa. Band 2: Mathematisches Wissen, pp. 213-224. Turnhout, Brepols (1998) 
5. Harborth, H.: Personal communication (2005)

6. Kemnitz, A.: Punktmengen mit ganzzahligen Abständen. Habilitationsschrift, TU Braunschweig (1988)

7. Kohnert, A., Kurz, S.: Integral point sets over $\mathbb{Z}_{n}^{m}$. Discrete Appl. Math. (2007, to appear)

8. Kreisel, T., Kurz, S.: List of integral hexagons in general position. http://www.wm.uni-bayreuth.de/ index.php?id=erdoes (2006)

9. Kurz, S.: Konstruktion und Eigenschaften ganzzahliger Punktmengen. PhD thesis, Bayreuth. Math. Schr. 76, Universität Bayreuth (2006)

10. Kurz, S.: On the characteristic of integral point sets in $\mathbb{E}^{m}$. Australas. J. Comb. 36, 241-248 (2006)

11. Kurz, S., Wassermann, A.: On the minimum diameter of plane integral point sets. Ars. Comb. (2007, submitted)

12. Noll, L., Bell, D.: $n$-clusters for $1<n<7$. Math. Comput. 53(187), 439-444 (1989) 